\title{
Cold molecular gas in cooling flow clusters of galaxies ${ }^{\star}$
}

\author{
P. Salomé and F. Combes
}

\begin{abstract}
Observatoire de Paris, LERMA, 61 Av. de l'Observatoire, 75014 Paris, France
\end{abstract}
Received 23 April 2002 / Accepted 9 August 2003

\begin{abstract}
The results of a CO line survey in central cluster galaxies with cooling flows are presented. Cold molecular gas is detected with the IRAM $30 \mathrm{~m}$ telescope, through $\mathrm{CO}(1-0)$ and $\mathrm{CO}(2-1)$ emission lines in 6-10 among 32 galaxies. The corresponding gas masses are between $3 \times 10^{8}$ and $4 \times 10^{10} M_{\odot}$. These results are in agreement with recent CO detections by Edge (2001). A strong correlation between the $\mathrm{CO}$ emission and the $\mathrm{H} \alpha$ luminosity is also confirmed. Cold gas exists in the center of cooling flow clusters and these detections may be interpreted as evidence of the long searched for very cold residual of the hot cooling gas.
\end{abstract}

Key words. galaxies: clusters: general - galaxies: cooling flows - galaxies: ISM

\section{Introduction}

Studies of X-ray emission of hot intra-cluster medium (ICM) have pointed out the high density of this gas in the central regions of many clusters. The derived timescales for radiative cooling in the center is much smaller than the Hubble time, and the ICM is predicted to condense and flow towards the cluster center (see Fabian 1994 for a review). The X-ray spectra show evidence of cooler gas in the center, through central drops of temperature. But the fate of the cooled gas still remains uncertain. The duration of the cooling flows is thought to be a significant fraction of the cluster life-time, since cooling flows are quite frequent in clusters. Estimated cooling rates of the order of $100 M_{\odot} / \mathrm{yr}$ and up to $1000 M_{\odot} / \mathrm{yr}$ implied that enormous quantities of material should have accumulated $\left(10^{11}\right.$ to $10^{12} M_{\odot}$ in a fraction of a Hubble time). But no resulting cold gas has been detected in molecular form until recently. Many efforts have been expended to detect this gas in emission or absorption, either in HI (Burns et al. 1981; Valentijn \& Giovanelli 1982; Shostak et al. 1983; McNamara et al. 1990; Dwarakanath et al. 1995) or in the CO molecule, see Grabelsky \& Ulmer (1990); McNamara et al. (1994); Antonucciet al. (1994); Braine \& Dupraz (1994); O'Dea et al. (1994). The intracluster medium is enriched in heavy elements with a metallicity of up to 0.3 solar making possible the formation of $\mathrm{CO}$ molecules. The first detection of $\mathrm{CO}$ emission has been made in Perseus A by Lazareff et al. (1989), but the corresponding $\mathrm{H}_{2}$ is not strongly identified as coming from the cooling flow rather than from

Send offprint requests to: P. Salomé,

e-mail: philippe.salome@obspm. fr)

* Tables 1-4 are also available at the CDS via anonymous

ftp to cdsarc.u-strasbg. fr (130.79.128.5)

http://cdsweb.u-strasbg.fr/cgi-bin/qcat?]/A+A/412/657 the galaxy itself. Recently, Edge (2001) reported to have found $\mathrm{CO}$ line emission in the central galaxy of sixteen extreme cooling flow clusters. Starbursts that may appear as a consequence of the gas condensation must produce a lot of young and hot stars. But the observed stellar luminosities are not bright enough to account for the high mass deposition rates of cooling flows. Although Chandra and XMM-Newton observations lead to reduced rates, the cold molecular gas masses observed in some cluster cores remain a small fraction of the gas cooled along the flow. The ICM is probably multi-phase (e.g. Ferland et al. 1994). A significant fraction of gas might be so cold (Pfenniger \& Combes 1994) that it could correspond to the high concentration of dark matter in clusters deduced from X-ray data and gravitational arcs (Durret et al. 1994; Wu \& Hammer 1993). Recently Lieu et al. (1996, 1999) and Mittaz et al. (1998) have detected large quantities of gas at intermediate temperature of $5 \times 10^{5} \mathrm{~K}$ in 5 clusters with the EUVE satellite (Extreme Ultraviolet Explorer). Since this phase is quite transient, the mass flow implied would be much larger than that of the cooling flow itself. Other processes must be at work, such as heating by shocks, or mixing layer mechanisms at the interface between a cold and hot phase (Bonamente et al. 2001). Also the detection of the near-infrared quadrupolar emission line $\mathrm{H}_{2}(1-0) \mathrm{S}(1)$ in central cluster galaxies with cooling flows (and their nondetection in similar control galaxies without cooling flows, e.g. Falcke et al. 1998) support the presence of molecular gas at temperature of $2000 \mathrm{~K}$ (Jaffe \& Bremer 1997; Edge et al. 2002; Wilman et al. 2002).

In this paper, we present our search for $\mathrm{CO}$ lines in 32 galaxies in the center of clusters, carried out in June and August 2001 with the IRAM 30 m telescope. We have found 6 clear detections and 4 hints of $\mathrm{CO}$ lines. In the next section we describe the instrumental conditions of our observations and 
the data reduction. We then present results and cold gas mass evaluations in Sect. 3. In Sects. 4 and 5 we discuss the possible significations of such large gas quantities when they are present and compare these measurements with other wavelength observations.

\section{Observations and data reduction}

The sample of sources was selected according to several criteria. First, we wanted to observe galaxies with important cooling flows, so we chose high deposition rates galaxies with $\dot{M}$ around or greater than $100 M_{\odot} / \mathrm{yr}$, see Peres et al. (1998), White et al. (1997), though these rates are certainly overestimated. Three non-cooling flow clusters (Abell 1668, Abell 1704 and Abell 2256) have also been observed and not detected in $\mathrm{CO}$ with the $30 \mathrm{~m}$ telescope. It is possible that the large gas flow produces massive stars ionizing the gas. The gas might also be cooling in ionizing shocks (optically luminous). Thus, the presence of large amounts of cooled gas could be accompanied by $\mathrm{H} \alpha$ emission as suggested in Edge (2001). Sources were then selected according to their $\mathrm{H}_{\alpha}$ luminosity when available (high luminosity of about $10^{42} \mathrm{erg} \mathrm{s}^{-1}$ from Crawford et al. 1999; Owen et al. 1995). The sample contains only relatively low-redshift $\mathrm{cD}$ galaxies $(z<0.25)$, for the sake of sensitivity. We gather data at other wavelengths, such as the far infra-red, when available, to be able to compare gas and dust emission. All observing parameters are summarized in Table 1. Observations were achieved with the IRAM $30 \mathrm{~m}$ millimeter-wave telescope at Pico Veleta, Spain in June and August 2001 in good weather conditions. We used four receivers simultaneously, centered two on the $\mathrm{CO}(1-0)$ and two on the $\mathrm{CO}(2-1)$ lines at $115 \mathrm{GHz}$ and $230 \mathrm{GHz}$. The beam of the telescope at these two frequencies is $22^{\prime \prime}$ and $13^{\prime \prime}$ respectively. Two backends were provided by the autocorrelator, with a $1.25 \mathrm{MHz}$ resolution on a $600 \mathrm{MHz}$ band width. The two other backends were the two $512 \mathrm{MHz}$ wide $1 \mathrm{MHz}$ filterbanks. These yield a total band of $\sim 1300 \mathrm{~km} \mathrm{~s}^{-1}$ at $2.6 \mathrm{~mm}$ and $\sim 650 \mathrm{~km} \mathrm{~s}^{-1}$ at $1.3 \mathrm{~mm}$. In addition, we used the $4 \mathrm{MHz}$ resolution filter-bank, providing a $1 \mathrm{GHz}$ band width, important for the $1.3 \mathrm{~mm}$ receivers (since it corresponds to $1300 \mathrm{~km} \mathrm{~s}^{-1}$ bandwidth also). Given the uncertainty in the central velocity of the CO line (some optically measured velocities being systematically displaced with respect to the galaxy systemic velocity), the expected width of a $\mathrm{cD}$ galaxy, and the required baseline to eliminate sinusoidal fluctuations, this wide band is necessary. The signals are expressed in main beam temperatures, since the sources are not expected to be extended and homogeneous. The main-beam efficiency of the $30 \mathrm{~m}$ is:

$\eta_{\mathrm{mb}}=T_{\mathrm{A}}^{*} / T_{\mathrm{mb}} \quad$ and $\quad \eta_{\mathrm{mb}}=B_{\mathrm{eff}} / F_{\mathrm{eff}}$

with $\eta_{\mathrm{mb}}=0.75 / 0.95$ at $115 \mathrm{GHz}$ and $0.52 / 0.91$ at $230 \mathrm{GHz}$ (cf. IRAM-30 m site http://www.iram.es/). The data were reduced with the CLASS package; spiky channels and bad scans were excised. After averaging all the raw spectra for each line of each source, linear baselines were subtracted and the spectra were Hanning smoothed. Assuming that a good sampling of the line requires at least five points, and assuming a typical $300 \mathrm{~km} \mathrm{~s}^{-1}$ line width, the data were smoothed to $55 \mathrm{~km} \mathrm{~s}^{-1}$ of spectral resolution, at 1.3 and $2.6 \mathrm{~mm}$, to gain more than a factor 3 in signal to noise. The $\mathrm{CO}$ emission lines were fitted with Gaussian profiles through the CLASS package. For non detections, CO intensity $\mathrm{I}_{\mathrm{CO}}$ upper limits at $3 \sigma$ were evaluated as in McNamara et al. (1994) by:

$I_{\mathrm{CO}}=3 \times \mathrm{rms} \times W_{\text {line }} \times\left(\frac{W_{\text {sm }}}{W_{\text {line }}}\right)^{1 / 2}$

where rms is the noise level, computed in the channel width $W_{\mathrm{sm}}\left(55 \mathrm{Km} \mathrm{s}^{-1}\right)$ and $W_{\text {line }}$ is the expected line bandwidth, typically $300 \mathrm{~km} \mathrm{~s}^{-1}$. The typical integration time was two hours for all galaxies. CO intensities were obtained by averaging the data of at least two different days, to prevent systematic effects (baseline ripples...). The detection criterion is the $\mathrm{CO}$ emission line maximum is at least three times the rms, in $55 \mathrm{~km} \mathrm{~s}^{-1}$ channels. When the signal to noise ratio was between 2 and $3 \mathrm{rms}$ we concluded to a hint of detection. Below $2 \mathrm{rms}$, upper limits were computed for the $\mathrm{CO}$ intensity. Results are presented in Tables 2 and 3. After this first analysis at each wavelength, we compare $I_{\mathrm{CO}(1-0)}$ and $I_{\mathrm{CO}(2-1)}$. A cold gas detection is claimed when $\mathrm{CO}$ is detected in the $\mathrm{CO}(1-0)$ and in the $\mathrm{CO}(2-1)$ lines simultaneously. Possible detections are defined by a detection in one line or by a hint of detection in both lines. Otherwise, we concluded to a non detection. For Abell 262, Abell 1068 and $\mathrm{Zw} 8193$, without $\mathrm{CO}(2-1)$ data, a detection is claimed because of a clear line detection in $\mathrm{CO}(1-0)$. With these criteria, we claim 6 detections, 4 possible detections, and 22 non detections. Detections made by Edge (2001) for Abell 1068 and RX J0821+07 are confirmed, with a good agreement of the derived hydrogen molecular gas masses. $\mathrm{CO}(1-0)$ emission line is also confirmed in Abell 262, but molecular gas mass deduced here is twice lower than in Edge (2001). That comes from the fact we identify a line with a smaller width. Three new values of $M_{\text {gas }}$ are found: Abell 646 for which cold gas mass is in agreement with Edge (2001) upper limit, Abell 1795 for which a large line width is found and consequently a molecular hydrogen mass higher than the upper limit deduced in Edge (2001) and PKS 0745-19 that was observed at a wrong frequency by Edge (2001).

$\mathrm{CO}$ intensities always correspond to areas deduced from Gaussian fits for detections and possible detections when a Gaussian fit was possible, but for the 22 no-detections, the $I_{\mathrm{CO}}$ are evaluated with formula (2). Table 4 show gas mass estimates as well as X-ray, optical, IR and radio data when available.

\section{Results}

\section{1. $\mathrm{H}_{2}$ mass evaluation from $\mathrm{CO}$ observations}

Since cold $\mathrm{H}_{2}$ is a symmetric molecule, the best tracer of cold molecular gas is the $\mathrm{CO}$ lines, from the most abundant molecule after $\mathrm{H}_{2}: \mathrm{CO} / \mathrm{H}_{2} \sim 6 \times 10^{-5}$. From standard (and empirical) calibrations, it is possible to deduce the interstellar $\mathrm{H}_{2}$ content from the integrated $\mathrm{CO}$ intensity $I_{\mathrm{CO}}\left(\mathrm{K} \mathrm{km} \mathrm{s}^{-1}\right)$ :

$I_{\mathrm{CO}}=\int T_{\mathrm{mb}}(\mathrm{CO}) \mathrm{d} V$

where $T_{\mathrm{mb}}(\mathrm{CO})$ is the main beam antenna temperature, obtained for the $\mathrm{CO}(1-0)$ line (cf. Sect. 2). Although the typical 
Table 1. This table presents the sample of cooling flow clusters of galaxies observed with the IRAM $30 \mathrm{~m}$ telescope. Central frequencies of the $\mathrm{CO}(1-0)$ and $\mathrm{CO}(2-1)$ lines observed, as the exposure time are indicated for each galaxy. Sources observed in the second run (August) are with indicated by a star. The others were observed during the first run (July). Several sources were not observed in $\mathrm{CO}(2-1)$ since the redshifted $J=2-1$ transition lines were out of the $30 \mathrm{~m}$ telescope receiver's band.

\begin{tabular}{|c|c|c|c|c|c|c|c|}
\hline Source & Redshift & RA (2000) & Decl (2000) & $\begin{array}{c}\text { Central Freq. } \\
\text { in } \mathrm{CO}(1-0)\end{array}$ & $\begin{array}{c}\text { Exposure } \\
\text { time (min) }\end{array}$ & $\begin{array}{l}\text { Central Freq. } \\
\text { in } \mathrm{CO}(2-1)\end{array}$ & $\begin{array}{l}\text { Exposure } \\
\text { time (min) }\end{array}$ \\
\hline A85* & 0.05567 & 004150.4 & -091811 & $109.19 \mathrm{GHz}$ & 152 & - & - \\
\hline Z235 & 0.08300 & 004352.1 & +242421 & $106.43 \mathrm{GHz}$ & 128 & $212.87 \mathrm{GHz}$ & 128 \\
\hline A $262^{*}$ & 0.01620 & 015246.5 & +360907 & $113.43 \mathrm{GHz}$ & 160 & - & - \\
\hline A291* & 0.19590 & 020143.1 & -021148 & $96.38 \mathrm{GHz}$ & 304 & - & - \\
\hline A496 & 0.03281 & 043337.8 & -131543 & $111.60 \mathrm{GHz}$ & 248 & $223.21 \mathrm{GHz}$ & 256 \\
\hline RX J0439+05 & 0.20800 & 043902.2 & +052044 & $95.42 \mathrm{GHz}$ & 300 & - & - \\
\hline PKS 0745-191 & 0.10280 & 074731.3 & -191740 & $104.52 \mathrm{GHz}$ & 304 & $209.04 \mathrm{GHz}$ & 376 \\
\hline A644* & 0.07040 & 081725.5 & -073044 & $107.69 \mathrm{GHz}$ & 144 & $215.37 \mathrm{GHz}$ & 88 \\
\hline RX J0821+07 & 0.11000 & 082102.4 & +075147 & $103.84 \mathrm{GHz}$ & 96 & $207.69 \mathrm{GHz}$ & 96 \\
\hline A646 & 0.12680 & 082209.6 & +470553 & $102.30 \mathrm{GHz}$ & 304 & $204.59 \mathrm{GHz}$ & 312 \\
\hline A780 & 0.05384 & 091805.7 & -120544 & $109.38 \mathrm{GHz}$ & 268 & $218.76 \mathrm{GHz}$ & 352 \\
\hline A1068* & 0.13860 & 104044.5 & +395711.1 & $101.23 \mathrm{GHz}$ & 180 & - & - \\
\hline A978 & 0.05425 & 102026.5 & -063136 & $109.34 \mathrm{GHz}$ & 96 & $218.67 \mathrm{GHz}$ & 96 \\
\hline A 1668 & 0.06368 & 130346.6 & +191618 & $108.37 \mathrm{GHz}$ & 80 & $216.73 \mathrm{GHz}$ & 80 \\
\hline A1795 & 0.06326 & 134852.4 & +263534 & $108.41 \mathrm{GHz}$ & 68 & $216.82 \mathrm{GHz}$ & 129 \\
\hline A2029 & 0.07795 & 151056.1 & +054441 & $106.93 \mathrm{GHz}$ & 112 & $213.86 \mathrm{GHz}$ & 168 \\
\hline MKW3s & 0.04531 & 152151.9 & +074232 & $110.27 \mathrm{GHz}$ & 144 & $220.54 \mathrm{GHz}$ & 144 \\
\hline A2146 & 0.23370 & 155613.8 & +66 2055 & $93.43 \mathrm{GHz}$ & 272 & - & - \\
\hline A $2142^{*}$ & 0.09037 & 155820.0 & +271402 & $105.71 \mathrm{GHz}$ & 128 & - & - \\
\hline A2147 & 0.03532 & 160217.0 & +155828 & $111.33 \mathrm{GHz}$ & 152 & $222.67 \mathrm{GHz}$ & 152 \\
\hline A 2151 & 0.03533 & 160435.8 & +174318 & $111.33 \mathrm{GHz}$ & 152 & $222.67 \mathrm{GHz}$ & 152 \\
\hline A2199 & 0.03035 & 162838.5 & +393306 & $111.87 \mathrm{GHz}$ & 128 & $223.74 \mathrm{GHz}$ & 120 \\
\hline Z8193* & 0.18290 & 171719.2 & +422700 & $97.44 \mathrm{GHz}$ & 304 & - & - \\
\hline A2261* & 0.2240 & 172227.1 & +320758 & $94.17 \mathrm{GHz}$ & 256 & - & - \\
\hline A2319* & 0.05459 & 192110.0 & +435644 & $109.30 \mathrm{GHz}$ & 136 & $218.60 \mathrm{GHz}$ & 88 \\
\hline CygA & 0.05607 & 195928.3 & +404402 & 109.15 GHz & 228 & $218.29 \mathrm{GHz}$ & 232 \\
\hline A 2462 & 0.07437 & 223911.4 & -172028 & $107.29 \mathrm{GHz}$ & 144 & $214.58 \mathrm{GHz}$ & 144 \\
\hline A2597 & 0.08520 & 232519.8 & -120726 & $106.22 \mathrm{GHz}$ & 144 & $212.43 \mathrm{GHz}$ & 144 \\
\hline A2626 & 0.05490 & 232630.6 & +210850 & 109.27 GHz & 136 & $218.54 \mathrm{GHz}$ & 140 \\
\hline A2634 & 0.03022 & 233829.5 & +270156 & $111.89 \mathrm{GHz}$ & 144 & $223.77 \mathrm{GHz}$ & 144 \\
\hline A2657 & 0.04023 & 234457.4 & +091134 & $110.81 \mathrm{GHz}$ & 208 & $221.62 \mathrm{GHz}$ & 208 \\
\hline A2665* & 0.05610 & 235050.6 & +060900 & $109.14 \mathrm{GHz}$ & 192 & $218.29 \mathrm{GHz}$ & 192 \\
\hline
\end{tabular}

molecular cloud is optically thick in the first CO lines of the $J$ ladder, the proportionality factor between the column density of molecular gas and integrated intensity is justified, since the observed signal is the emission sum of many clouds in the beam, and these clouds have a small filling factor, when spatial and velocity volume is considered. We adopt here the conversion factor commonly used for $N\left(\mathrm{H}_{2}\right)$ in molecule $/ \mathrm{cm}^{2}$ unit:

$N\left(\mathrm{H}_{2}\right)=2.3 \times 10^{20} I_{\mathrm{CO}}$

From this equation the mass of molecular hydrogen, contained in one beam, in $M_{\odot}$ is:

$M\left(\mathrm{H}_{2}\right)=2.95 \times 10^{-19} I_{\mathrm{CO}} \theta^{2} D^{2} \frac{N\left(\mathrm{H}_{2}\right)}{I_{\mathrm{CO}}}$ where $I_{\mathrm{CO}}$ is the integrated intensity in $\mathrm{K} \mathrm{km} \mathrm{s}^{-1}, \theta$ is the beamsize of the telescope in arcsec, and $D$ is the distance of the galaxy in Mpc, determined with a Hubble constant $H_{0}=$ $70 \mathrm{~km} \mathrm{~s}^{-1} \mathrm{Mpc}^{-1}$. This converting factor is in agreement with the one used in Edge (2001) and in previous CO observations of cluster cores but gas mass estimations are slightly lower here because we took $H_{0}=70 \mathrm{~km} \mathrm{~s}^{-1} \mathrm{Mpc}^{-1}$. It is important to notice the $I_{\mathrm{CO}} / N\left(\mathrm{H}_{2}\right)$ conversion factor has been first calibrated in the solar neighborhood. To use this value implies intra-cluster medium is assumed to behave like the Galactic interstellar matter near the sun, in particular with the solar metallicity. But the intracluster medium has subsolar metallicity. So this conversion factor is likely to underestimate the mass of molecular hydrogen. A standard factor 1.36 taking into account $\mathrm{He}$ 
Table 2. Summary of observational data for the two runs. Lines characteristics are presented, spectra are shown in Fig. 8. $I_{\mathrm{CO}}$ (Col. 8) were evaluated from a Gaussian fit of the $\mathrm{CO}(1-0)$ and $\mathrm{CO}(2-1)$ lines for detections and hints of detections. $I_{\mathrm{CO}}$ upper limits were evaluated by equation 2 for non-detections. A detection is asserted in one transition line when the peak of the Gaussian fit is above three times the rms (in $55 \mathrm{~km} \mathrm{~s}^{-1}$ channels) and a hint of detection when it is between two and three times the rms. To claim a cold molecular gas detection, we require a detection in both transition lines. A possible detection was claimed when was present a detection in one transition line or a hint of detection in both lines, unless the line appear clearly in one transition only (Abell 262, Abell 1068, Zw8193).

\begin{tabular}{lccccccc}
\hline \hline \multicolumn{1}{c}{ Source } & Line & $\begin{array}{c}\text { Peak } \\
\mathrm{mK}\end{array}$ & $\begin{array}{c}\text { Rms } \\
\mathrm{mK}\end{array}$ & $\begin{array}{c}\text { Line } \\
\text { detection }\end{array}$ & $\begin{array}{c}\text { Line } \\
\text { position }\left(\mathrm{km} \mathrm{s}^{-1}\right)\end{array}$ & $\begin{array}{c}\text { Line } \\
\text { width }\left(\mathrm{km} \mathrm{s}^{-1}\right)\end{array}$ & $\begin{array}{c}I_{\mathrm{CO}} \\
\mathrm{K} \mathrm{km} \mathrm{s}^{-1}\end{array}$ \\
\hline A85* & $\mathrm{CO}(1-0)$ & - & 0.9 & no & - & $300^{\star}$ & $\leq 0.35$ \\
Z235 & $\mathrm{CO}(1-0)$ & $1.9 \pm 0.2$ & 0.8 & hint & $-258 \pm 48$ & $318 \pm 103$ & $0.66 \pm 0.19$ \\
& $\mathrm{CO}(2-1)$ & - & 1.7 & no & - & $300^{\star}$ & $\leq 0.65$ \\
A262* & $\mathrm{CO}(1-0)$ & $2.9 \pm 0.5$ & 0.8 & yes & $31 \pm 24$ & $346 \pm 47$ & $1.05 \pm 0.14$ \\
A291* & $\mathrm{CO}(1-0)$ & - & 0.9 & no & - & $300^{\star}$ & $\leq 0.35$ \\
A496 & $\mathrm{CO}(1-0)$ & $1.5 \pm 0.5$ & 0.7 & hint & $382 \pm 39$ & $311 \pm 73$ & $0.49 \pm 0.12$ \\
& $\mathrm{CO}(2-1)$ & $3.0 \pm 0.7$ & 1.1 & hint & $114 \pm 29$ & $249 \pm 52$ & $0.80 \pm 0.18$ \\
RX J0439+05 & $\mathrm{CO}(1-0)$ & - & 0.72 & no & - & $300^{\star}$ & $\leq 0.28$ \\
PKS 0745-191 & $\mathrm{CO}(1-0)$ & $2.0 \pm 0.3$ & 0.6 & yes & $18 \pm 29$ & $221 \pm 58$ & $0.47 \pm 0.11$ \\
& $\mathrm{CO}(2-1)$ & $11.2 \pm 0.8$ & 2.1 & yes & $-45 \pm 15$ & $215 \pm 41$ & $2.57 \pm 0.38$ \\
A644* & $\mathrm{CO}(1-0)$ & $1.1 \pm 0.4$ & 0.7 & no & $-10 \pm 55$ & $260 \pm 77$ & $\leq 0.27$ \\
& $\mathrm{CO}(2-1)$ & - & 3.1 & no & - & $300^{\star}$ & $\leq 1.12$ \\
RX J0821+07 & $\mathrm{CO}(1-0)$ & $8.9 \pm 0.6$ & 1.1 & yes & $-2 \pm 8$ & $135 \pm 21$ & $1.28 \pm 0.16$ \\
& $\mathrm{CO}(2-1)$ & $9.9 \pm 1.5$ & 2.9 & yes & $5 \pm 34$ & $270 \pm 81$ & $2.86 \pm 0.60$ \\
A646 & $\mathrm{CO}(1-0)$ & $1.5 \pm 0.1$ & 0.5 & yes & $105 \pm 39$ & $376 \pm 121$ & $0.62 \pm 0.15$ \\
& $\mathrm{CO}(2-1)$ & $1.9 \pm 1.0$ & 1.6 & no & $46 \pm 54$ & $346 \pm 123$ & $\leq 0.62$ \\
A780 & $\mathrm{CO}(1-0)$ & $1.8 \pm 0.5$ & 0.6 & yes & $219 \pm 31$ & $439 \pm 68$ & $0.80 \pm 0.11$ \\
& $\mathrm{CO}(2-1)$ & - & 1.8 & no & - & $300^{\star}$ & $\leq 0.69$ \\
A978 & $\mathrm{CO}(1-0)$ & $1.8 \pm 0.7$ & 1.0 & no & $-128 \pm 49$ & $221 \pm 87$ & $\leq 0.39$ \\
& $\mathrm{CO}(2-1)$ & - & 2.1 & no & - & $300^{\star}$ & $\leq 0.80$ \\
A1068* & $\mathrm{CO}(1-0)$ & $10.1 \pm 0.3$ & 0.5 & yes & $-45 \pm 4$ & $249 \pm 10$ & $2.66 \pm 0.1$ \\
A1668 & $\mathrm{CO}(1-0)$ & - & 1.2 & no & - & $300^{\star}$ & $\leq 0.46$ \\
& $\mathrm{CO}(2-1)$ & $2.4 \pm 1.6$ & 1.7 & no & $9 \pm 64$ & $281 \pm 125$ & $\leq 0.65$ \\
A1795 & $\mathrm{CO}(1-0)$ & $3.4 \pm 0.7$ & 0.9 & yes & $-190 \pm 28$ & $405 \pm 56$ & $1.47 \pm 0.19$ \\
& $\mathrm{CO}(2-1)$ & $6.1 \pm 1.0$ & 1.8 & yes & $-128 \pm 31$ & $500 \pm 65$ & $3.26 \pm 0.39$ \\
\hline
\end{tabular}

contribution is also used in the gas mass estimation: $M_{\mathrm{gas}}=$ $1.36 M\left(\mathrm{H}_{2}\right)$. The derived $M_{\text {gas }}$ values, displayed in Table 4 , are between $\sim 10^{8}$ and $\sim 10^{10} M_{\odot}$. These masses, together with upper limits, are plotted in Fig. 1 for all sources, as a function of redshift. The curve represents the $M_{\text {gas }}$ detection limit of the IRAM $30 \mathrm{~m}$ telescope for the $\mathrm{CO}(1-0)$ line, assuming a typical $300 \mathrm{~km} \mathrm{~s}^{-1}$ linewith and a temperature detection limit of $0.5 \mathrm{mK}$, in agreement with our noise level.

\section{2. $\mathrm{CO}$ detections}

Abell 262 has also been detected by Edge (2001). Current values are compatible with these measurements and confirm this detection even if $\mathrm{CO}(2-1)$ data were not good enough in the second run of August 2001 to detect a line. This galaxy contains a central radio source according to Peres et al. (1998) and show low luminosity optical lines, see Crawford et al. (1999).

PKS 0745-191 is a 0.1028 redshifted galaxy already observed in $\mathrm{CO}$ by Edge (2001). In present observations, a CO(1$0)$ line is detected three times above the rms. A simultaneous detection in the $\mathrm{CO}(2-1)$ band with a signal to noise better then five seems to confirm the presence of molecular gas. This galaxy is supposed to contain a large cooling flow with mass deposition rates around $1000 M_{\odot} / y r$ according

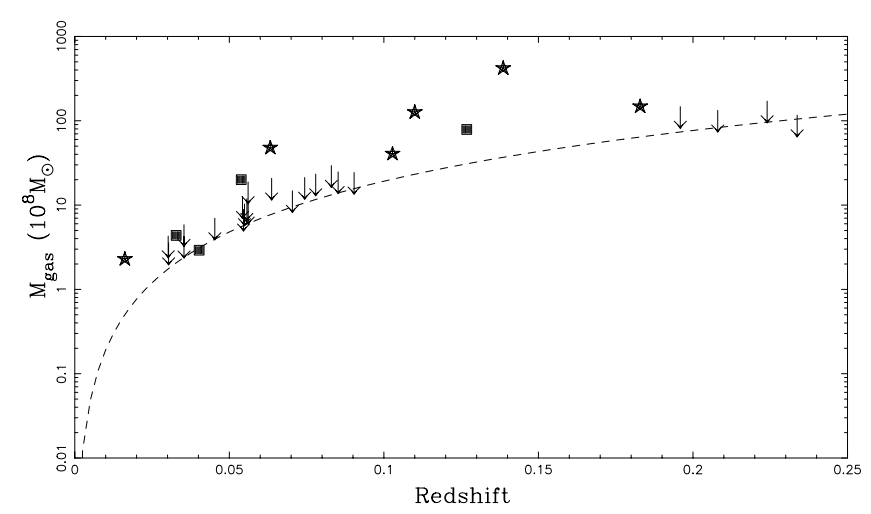

Fig. 1. $M_{\text {gas }}$ in $10^{8} M_{\odot}$ deduced from CO observations, versus the galaxy redshift. Filled stars are detections, filled square hints of detection and arrows are upper limits. The dashed line represents the molecular mass limit than can be deduced from $\mathrm{CO}$ observations with the IRAM $30 \mathrm{~m}$ telescope, in $2 \mathrm{~h}$ integration time.

to Peres et al. (1998), Allen (2000). Strong optical emission lines have also been detected in PKS 0745-191. This galaxy is the site of an important excitation mechanism. Besides it is a powerful radio source with an amorphous and filamentary morphology, see Baum \& O’Dea (1991). Recently, Donahue et al. (2000) have mapped kpc-size filaments in 
Table 3. Summary of observational data for the two runs. Lines characteristics (Table 2 continuation).

\begin{tabular}{|c|c|c|c|c|c|c|c|}
\hline Source & Line & $\begin{array}{l}\text { Peak } \\
\text { mK }\end{array}$ & $\begin{array}{c}\mathrm{Rms} \\
\mathrm{mK}\end{array}$ & $\begin{array}{c}\text { Line } \\
\text { detection }\end{array}$ & $\begin{array}{c}\text { Line } \\
\text { position }\left(\mathrm{km} \mathrm{s}^{-1}\right)\end{array}$ & $\begin{array}{c}\text { Line } \\
\text { width }\left(\mathrm{km} \mathrm{s}^{-1}\right)\end{array}$ & $\begin{array}{c}I_{\mathrm{CO}} \\
\mathrm{K} \mathrm{km} \mathrm{s}^{-1}\end{array}$ \\
\hline \multirow[t]{2}{*}{ A2029 } & $\mathrm{CO}(1-0)$ & $1.1 \pm 0.5$ & 0.9 & no & $-68 \pm 87$ & $445 \pm 168$ & $\leq 0.35$ \\
\hline & $\mathrm{CO}(2-1)$ & - & 1.6 & no & - & $300^{\star}$ & $\leq 0.62$ \\
\hline \multirow[t]{2}{*}{ MKW3s } & $\mathrm{CO}(1-0)$ & $1.3 \pm 0.4$ & 0.8 & no & $-133 \pm 60$ & $347 \pm 100$ & $\leq 0.31$ \\
\hline & $\operatorname{CO}(2-1)$ & - & 1.0 & no & - & $300^{\star}$ & $\leq 0.39$ \\
\hline A2146 & $\mathrm{CO}(1-0)$ & - & 0.5 & no & - & $300^{\star}$ & $\leq 0.19$ \\
\hline A2142* & $\mathrm{CO}(1-0)$ & - & 0.7 & no & - & $300^{\star}$ & $\leq 0.27$ \\
\hline \multirow[t]{2}{*}{ A2147 } & $\mathrm{CO}(1-0)$ & - & 1.1 & no & - & $300^{\star}$ & $\leq 0.42$ \\
\hline & $\mathrm{CO}(2-1)$ & - - & 1.4 & no & - & $300^{\star}$ & $\leq 0.54$ \\
\hline \multirow[t]{2}{*}{ A2151 } & $\mathrm{CO}(1-0)$ & $2.0 \pm 0.3$ & 0.8 & hint & $341 \pm 47$ & $133 \pm 95$ & $0.29 \pm 0.14$ \\
\hline & $\mathrm{CO}(2-1)$ & - & 1.5 & no & - & $300^{\star}$ & $\leq 0.58$ \\
\hline \multirow[t]{2}{*}{ A2199 } & $\mathrm{CO}(1-0)$ & $1.6 \pm 0.4$ & 0.9 & no & $109 \pm 55$ & $395 \pm 123$ & $\leq 0.35$ \\
\hline & $\mathrm{CO}(2-1)$ & $3.1 \pm 0.6$ & 1.2 & hint & $265 \pm 28$ & $204 \pm 61$ & $0.67 \pm 0.18$ \\
\hline Z8193* & $\mathrm{CO}(1-0)$ & $2.1 \pm 0.4$ & 0.4 & yes & $-14 \pm 20$ & $242 \pm 65$ & $0.55 \pm 0.1$ \\
\hline A2261* & $\mathrm{CO}(1-0)$ & - & 0.8 & no & - & $300^{\star}$ & $\leq 0.31$ \\
\hline \multirow[t]{2}{*}{ A2319* } & $\mathrm{CO}(1-0)$ & - & 0.7 & no & - & $300^{\star}$ & $\leq 0.27$ \\
\hline & $\mathrm{CO}(2-1)$ & - & 3.4 & no & - & $300^{\star}$ & $\leq 1.3$ \\
\hline \multirow[t]{2}{*}{ CygA } & $\mathrm{CO}(1-0)$ & $3.6 \pm 0.3$ & 1.4 & hint & $203 \pm 24$ & $153 \pm 46$ & $0.59 \pm 0.18$ \\
\hline & $\mathrm{CO}(2-1)$ & $2.1 \pm 0.5$ & 1.4 & no & $-83 \pm 65$ & $336 \pm 207$ & $\leq 0.54$ \\
\hline \multirow[t]{2}{*}{ A2462 } & $\mathrm{CO}(1-0)$ & - & 0.9 & no & - & $300^{\star}$ & $\leq 0.35$ \\
\hline & $\mathrm{CO}(2-1)$ & - & 2.7 & no & - & $300^{\star}$ & $\leq 1.0$ \\
\hline \multirow[t]{2}{*}{ A2597 } & $\mathrm{CO}(1-0)$ & $1.1 \pm 0.3$ & 0.8 & no & $-10 \pm 55$ & $260 \pm 77$ & $0.29 \pm 0.11$ \\
\hline & $\mathrm{CO}(2-1)$ & - & 2.3 & no & - & $300^{\star}$ & $\leq 0.89$ \\
\hline \multirow[t]{2}{*}{ A2626 } & $\mathrm{CO}(1-0)$ & $1.7 \pm 0.2$ & 0.8 & hint & $-161 \pm 42$ & $293 \pm 103$ & $0.52 \pm 0.15$ \\
\hline & $\mathrm{CO}(2-1)$ & - & 1.4 & no & - & $300^{\star}$ & $\leq 0.54$ \\
\hline \multirow[t]{2}{*}{ A2634 } & $\mathrm{CO}(1-0)$ & $2.9 \pm 0.4$ & 1.1 & hint & $488 \pm 62$ & $459 \pm 137$ & $1.44 \pm 0.38$ \\
\hline & $\mathrm{CO}(2-1)$ & - & 2.2 & no & - & $300^{\star}$ & $\leq 0.85$ \\
\hline \multirow[t]{2}{*}{ A2657 } & $\mathrm{CO}(1-0)$ & $3.0 \pm 0.03$ & 0.8 & yes & $147 \pm 13$ & $69 \pm 50$ & $0.22 \pm 0.080$ \\
\hline & $\mathrm{CO}(2-1)$ & - & 0.8 & no & - & $300^{\star}$ & $\leq 0.31$ \\
\hline \multirow[t]{2}{*}{ A2665* } & $\mathrm{CO}(1-0)$ & $1.5 \pm 0.2$ & 0.8 & no & $-273 \pm 40$ & $193 \pm 82$ & $\leq 0.31$ \\
\hline & $\mathrm{CO}(2-1)$ & - & 1.6 & no & - & $300^{\star}$ & $\leq 0.62$ \\
\hline
\end{tabular}

vibrationally-excited $\mathrm{H}_{2}$ in the cores of galaxies centers of cooling flows, like PKS 0745-191, with high spatial resolution. They have also found dust lanes which are optically thick to $1.6 \mu \mathrm{m}$ emission. These dust lanes are confined to the central few kpcs.

The cD galaxy RX J0821+07 has been detected in CO by Edge (2001). This relatively easy detection is confirmed here in both wavelength and $\mathrm{CO}$ intensities are compatible with previous ones. Optical images taken with the AAT and Hubble Space Telescope by Bayer-Kim et al. (2002) show that the central galaxy is embedded in a luminous and extended line-emitting nebula coincident with a bright excess of X-ray emission imaged by Chandra.

Abell 1068 detected by Edge (2001) is confirmed here. The $\mathrm{H}_{2}$ mass deduced is the highest we found among detections. This galaxy show strong optical lines and large dust mass in comparison with other detections. It is also a powerful IRAS source with a $650 \mathrm{~m}$ Jy flux at $60 \mu \mathrm{m}$.

Abell 1795 has been observed by Braine \& Dupraz (1994) who did not detect CO line. Edge (2001) found a marginal detection. This detection is confirmed here in the two bands. But line widths deduced from Gaussian fits are quite different in the two wavelengths, so we cannot exclude the possibility of very high velocity molecular clouds. This galaxy is known as a radio source see David et al. (1993). An optical filament has been detected in $\mathrm{H}_{\alpha}$ by Cowie et al. (1983). According to them, some of the filaments observed in Abell 1795 seem to be concentrated and coming from the galaxy whereas fainter extended filaments are surrounding the galaxy. The question about their origin and their link with the cooling flows is not clearly determined. Mapping cold gas will allow to better understand the spatial structure of the cooling material and to know if the $\mathrm{CO}$ is along the filaments or in the galaxy, Salomé \& Combes (2003, in prep).

Zwicky 8193 is a strong optical line emitter. Only $\mathrm{CO}(1-0)$ was observed here. The molecular gas mass deduced agree with the value derived in Edge (2001). Zwicky 8193 is a complex system and we refer to Edge (2001) and Edge et al. (2002) for the discussion.

\subsection{Hints of detection}

We consider 4 galaxies of our sample to be possible $\mathrm{CO}$ emitters according to criteria defined above. Nevertheless $\mathrm{CO}$ emission lines here are fainter than the previous ones, with values reaching half a $\mathrm{K} \mathrm{km} \mathrm{s}^{-1}$.

Abell 496 is a cD galaxy. A possible line is seen in the two bands, but signal to noise ratio between 2 and 3 is not 
sufficient to claim a detection. Much time has been dedicated to this radio source, see for example Peres et al. (1998), to deduce a small upper limit of $\mathrm{H}_{2}$ mass. Faint optical $\mathrm{H}_{\alpha}$ line have been observed in this galaxy also emitting in X-ray, see David et al. (1993). Nevertheless we deduce here a new upper limit in molecular gas mass.

We also assert a possible detection in Abell 646, even if no $\mathrm{CO}(2-1)$ line is seen, because of the clear shape of the $\mathrm{CO}(1-0)$ line detected just above three time the rms. Moreover, Edge (2001) asserted to have a marginal detection of this galaxy. So it would be interesting to confirm this detection.

Abell 780 (Hydra A) is a very powerful radio source that had already been observed through millimetric wavelength, see for example O'Dea et al. (1994b). This much studied source is here at the limit of detection in $\mathrm{CO}(1-0)$ and not seen in $\mathrm{CO}(2-1) . M_{\text {gas }}$ upper limit deduced is in agreement with the evaluation made by Edge (2001), but no clear detection can be claimed.

The cD Abell 2657 galaxy with optical emission lines was not detected in $\mathrm{CO}(2-1)$. Faint possible $\mathrm{CO}(1-0)$ line is present and a new upper limit in $M_{\text {gas }}$ is derived, but more observations are required.

\subsection{Upper limits}

Many of the selected galaxies are not detected. It is possible, these cooling flow clusters of galaxies contains cold gas with lines still too weak to be detectable with the actual IRAM $30 \mathrm{~m}$ telescope sensitivity.

\section{Discussion}

\subsection{Origin of the cold gas}

Recent X-ray observations by Chandra, see Fabian (2002), Voigt et al. (2002) and XMM-Newton (Peterson et al. 2001; Tamura et al. 2001) have confirmed the presence of radial gradients in temperature in the cores of several clusters of galaxies. Even if results from the high spectral resolution Reflection Grating Spectrometer (RGS) on XMM-Newton do not show evidence (from Fe XVII) for gas cooling at temperature lower than 1-2 kev, millimetric emission of a cold gas component is detected in the center of several galaxy clusters. Added to recent data from Edge (2001), new detections of molecular gas in cooling flow galaxies is of great interest. But questions persist on the origin of this cold phase and its place in a gas infall scenario.

\subsection{Optically thick cold clouds}

In an optically thick medium which is the case here, the $\mathrm{CO}(2-1) / \mathrm{CO}(1-0)$ ratio should be about or less than one, if we assume the same excitation temperature for the two $\mathrm{CO}$ energy levels. In Fig. 2 is plotted the $\mathrm{CO}(2-1)$ versus $\mathrm{CO}(1-$ 0 ) intensity (in $\mathrm{K} \mathrm{km} \mathrm{s}^{-1}$ ) for the galaxies observed here. The straight line indicates their equality. $\mathrm{CO}(2-1)$ intensities have been multiplied by a beam correcting factor $\sim 4$ and by the relative beam efficiencies $(0.52 / 0.91) /(0.75 / 0.95)=0.72$ to be

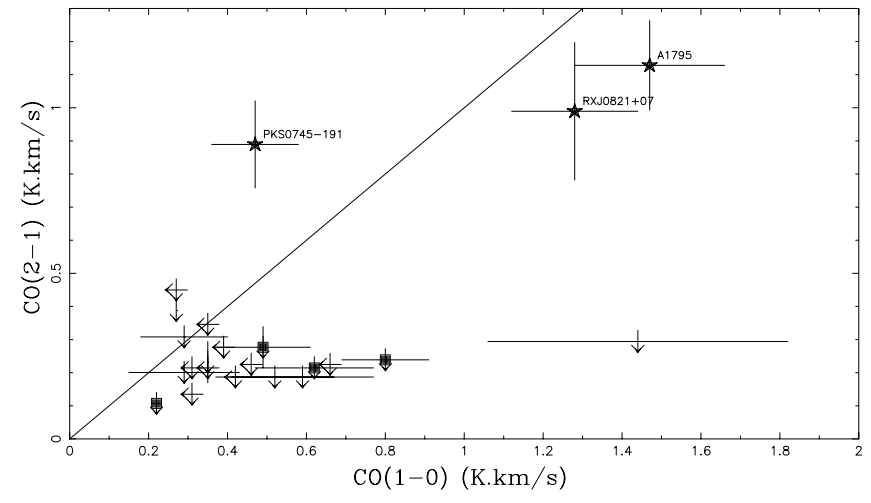

Fig. 2. $\mathrm{CO}(2-1)$ versus $\mathrm{CO}(1-0)$ corrected from the effect of different beam sizes. The straight line corresponds to line emission equality.

compared to the $\mathrm{CO}(1-0)$ ones. The preliminary plot indicates that the $\mathrm{CO}(2-1)$ line is in fact lower than the $\mathrm{CO}(1-0)$ one; this is in general the case for sub-thermally excited gas, in nearby galaxies (e.g. Braine \& Combes 1992). The CO lines ratio are consistent with an optically thick gas. The medium considered here is certainly far more complex, probably inhomogeneous and multi-phase. It might be a mixing of diffuse gas and denser clumps, and the diffuse medium might be dominating the emission, while thick and small clouds could enclose a larger quantity of hydrogen mass than estimated.

\subsection{Origin of cold gas}

If the molecular gas is formed by the cooling flow, we should see a correlation between the detected cold gas masses and the X-ray determined mass deposition rate, as shown by Edge (2001). But there is a quite large dispersion in the $\dot{M}_{\mathrm{X}}$ values because of the different methods used in the literature, see Grabelsky \& Ulmer (1990), Bregman et al. (1990), White et al. (1997), Peres et al. (1998) or Allen (2000). To test this, we have compared cold gas masses found here with mass deposition rates evaluated thanks to an Einstein Observatory Xray image deprojection analysis made by White et al. (1997), see Fig. 3. The comparison is also done with $\dot{M}$ issue from a ROSAT observatory spatial analysis by Peres et al. (1998) as shown in Fig. 4. These two samples of mass deposition rates, evaluated from X-ray data, have been chosen because they contain the largest number of sources in common with the clusters observed here in $\mathrm{CO}$. For a correlation trend to be relevant, the aim was (i) to have a high number of sources observed in both $\mathrm{CO}$ and X-ray, with regards to the faint detection level in $\mathrm{CO}$ and (ii) to compare $M\left(\mathrm{H}_{2}\right)$ with $\dot{M}$ derived from one method only (with the same criteria for all sources). We can see a trend of correlation do appear, even if there are very few data points. This confirms the relation between the mass of the cold component and the mass deposition rate already noticed in Edge (2001).

Galaxies for which measurements have been possible lie close to $M_{\text {gas }}=1 \% \times \dot{M} \times 1$ Gyr (large symbols). Then, assuming that simple models of a multiphase flow would lead to an integrated mass deposition profile of the form $\dot{M}(<r) \propto r^{\alpha}$, with $\alpha \sim 1$. We have re-evaluated what would be the $\dot{M}$ 
Table 4. Derived parameters of the observed sources. Optical line luminosities are from Crawford et al. (1999). Mass deposition rates and cooling radius are from (1) Peres et al. (1998) and (2) White et al. (1997). Dust masses are evaluated from $60 \mu \mathrm{m}$ data compiled in Edge (2001), assuming $T_{\text {dust }}=35 \mathrm{~K}$.

\begin{tabular}{|c|c|c|c|c|c|c|c|c|}
\hline Source & $\begin{array}{c}M\left(\mathrm{H}_{2}\right) \\
\left(M_{\odot}\right)\end{array}$ & $\begin{array}{l}M_{\text {dust }} \\
\left(M_{\odot}\right)\end{array}$ & $\begin{array}{l}L\left(\mathrm{H}_{\alpha}\right) \\
(\mathrm{erg} / \mathrm{s})\end{array}$ & $\begin{array}{c}\dot{M}^{(1)} \\
\left(M_{\odot} / \mathrm{yr}\right)\end{array}$ & $\begin{array}{c}r_{\text {cool }}^{(1)} \\
(\mathrm{kpc})\end{array}$ & $\begin{array}{c}\dot{M}^{(2)} \\
\left(M_{\odot} / \mathrm{yr}\right)\end{array}$ & $\begin{array}{c}r_{\text {cool }}^{(2)} \\
(\mathrm{kpc})\end{array}$ & $\begin{array}{c}\text { Flux }(1.4 \mathrm{GHz}) \\
(\mathrm{m} \mathrm{Jy})\end{array}$ \\
\hline A262 & $2.3 \pm 0.3 \times 10^{8}$ & $8.7 \times 10^{6}$ & $6.0 \times 10^{39}$ & 27 & 104 & 10 & 67 & 131 \\
\hline pks 0745-191 & $4.0 \pm 0.9 \times 10^{9}$ & $3.5 \times 10^{7}$ & $1.4 \times 10^{42}$ & 1038 & 214 & 579 & 177 & 2370 \\
\hline rx j0821+07 & $1.3 \pm 0.2 \times 10^{10}$ & $4.2 \times 10^{7}$ & $3.0 \times 10^{41}$ & - & - & - & - & - \\
\hline A1068 & $4.2 \pm 0.2 \times 10^{10}$ & $1.4 \times 10^{8}$ & $1.7 \times 10^{42}$ & - & - & - & - & - \\
\hline A1795 & $4.8 \pm 0.6 \times 10^{9}$ & $6.7 \times 10^{7}$ & $1.1 \times 10^{41}$ & 381 & 177 & 321 & 181 & 930 \\
\hline Z8193 & $1.5 \pm 0.3 \times 10^{10}$ & $3.8 \times 10^{7}$ & $1.5 \times 10^{42}$ & - & - & - & - & - \\
\hline A496 & $4.3 \pm 1.0 \times 10^{8}$ & - & $3.4 \times 10^{42}$ & 95 & 110 & 134 & 138 & - \\
\hline A646 & $7.9 \pm 0.2 \times 10^{9}$ & $2.5 \times 10^{7}$ & $1.6 \times 10^{41}$ & - & - & - & - & - \\
\hline A780 & $2.0 \pm 0.3 \times 10^{9}$ & $3.0 \times 10^{7}$ & $1.6 \times 10^{41}$ & 262 & 162 & 222 & 170 & 40800 \\
\hline A2657 & $2.9 \pm 0.1 \times 10^{8}$ & - & - & - & - & 44 & 101 & - \\
\hline A85 & $\leq 8.8 \times 10^{8}$ & - & - & 107 & 93 & 108 & 131 & 58 \\
\hline $\mathrm{Z} 235$ & $\leq 2.5 \times 10^{9}$ & - & - & - & - & - & - & - \\
\hline A291 & $\leq 1.1 \times 10^{10}$ & $84.9 \times 10^{7}$ & $4.6 \times 10^{41}$ & - & - & - & - & - \\
\hline A644 & $\leq 1.1 \times 10^{9}$ & - & - & 189 & 141 & 136 & 111 & - \\
\hline rx j0439+05 & $\leq 9.8 \times 10^{9}$ & $5.1 \times 10^{7}$ & $1.1 \times 10^{42}$ & - & - & - & - & - \\
\hline A978 & $\leq 9.3 \times 10^{8}$ & - & $2.0 \times 10^{40}$ & - & - & - & - & - \\
\hline A1668 & $\leq 1.5 \times 10^{9}$ & - & $1.2 \times 10^{41}$ & - & - & - & - & - \\
\hline A2029 & $\leq 1.7 \times 10^{9}$ & - & $8.0 \times 10^{39}$ & 555 & 186 & 431 & 192 & 550 \\
\hline MKW3s & $\leq 5.2 \times 10^{8}$ & - & $5.0 \times 10^{39}$ & 175 & 171 & 132 & 158 & - \\
\hline A2146 & $\leq 8.6 \times 10^{9}$ & $8.8 \times 10^{7}$ & $1.4 \times 10^{42}$ & - & - & - & - & - \\
\hline A2142 & $\leq 1.8 \times 10^{9}$ & - & - & 350 & 150 & 369 & 172 & - \\
\hline A2147 & $\leq 4.3 \times 10^{8}$ & - & $7.0 \times 10^{39}$ & - & - & - & - & - \\
\hline A2151 & $\leq 3.1 \times 10^{8}$ & - & $5.8 \times 10^{40}$ & - & - & 166 & 146 & - \\
\hline A2199 & $\leq 2.6 \times 10^{8}$ & - & $3.5 \times 10^{40}$ & 154 & 143 & 97 & 124 & 3700 \\
\hline A2261 & $\leq 1.3 \times 10^{10}$ & - & - & - & 20 & 53 & - & - \\
\hline A2319 & $\leq 6.6 \times 10^{8}$ & - & $1.0 \times 10^{41}$ & - & - & - & - & - \\
\hline CygA & $\leq 1.4 \times 10^{9}$ & - & $6.5 \times 10^{42}$ & 244 & 135 & 242 & 167 & - \\
\hline A2462 & $\leq 1.6 \times 10^{9}$ & - & $5.8 \times 10^{40}$ & - & - & - & - & - \\
\hline A2597 & $\leq 1.8 \times 10^{9}$ & $8.4 \times 10^{7}$ & $5.2 \times 10^{41}$ & 271 & 152 & - & - & 1880 \\
\hline A2626 & $\leq 7.6 \times 10^{8}$ & - & $3.3 \times 10^{40}$ & - & - & 53 & 114 & - \\
\hline A2634 & $\leq 3.2 \times 10^{8}$ & - & $3.7 \times 10^{40}$ & - & - & - & - & 7657 \\
\hline A2665 & $\leq 7.9 \times 10^{8}$ & - & $6.0 \times 10^{39}$ & - & - & - & - & - \\
\hline
\end{tabular}

inside the $30 \mathrm{~m}$ telescope radius with a simple scaling by the cooling radius to the $\mathrm{CO}$ radius ratio. The correlation still appear but the cold gas masses detected are now close to $M_{\text {gas }}=\dot{M} \times 10 \% \times 1$ Gyr (small symbols with gray background). These mass deposition rates have probably been overestimated by about a factor 5-10, see McNamara et al. (2002), as suggested by the recent X-ray observations by Chandra and XMM-Newton (e.g. Abell 1795 in Fabian 2002; Abell 2199 in Johnstone et al. 2002 or Abell 496 in Dupke \& White 2003). Taking into account the uncertainty on the conversion factor between $\mathrm{H}_{2}$ and $\mathrm{CO}$, as discussed above, the correlation is in accordance with a cooling scenario in which hot gas lead to cold substructures at rate deduced by X-ray observations and detected here in $\mathrm{CO}$ (for an assumed age of the cooling is a few Gyr in the central regions).

But many galaxies in cooling flow clusters observed here do not show CO emission lines. Given the faint emission temperature, it is possible that the cold gas is present but its radiation is below the detection limit. An alternative is the gas is not cooled

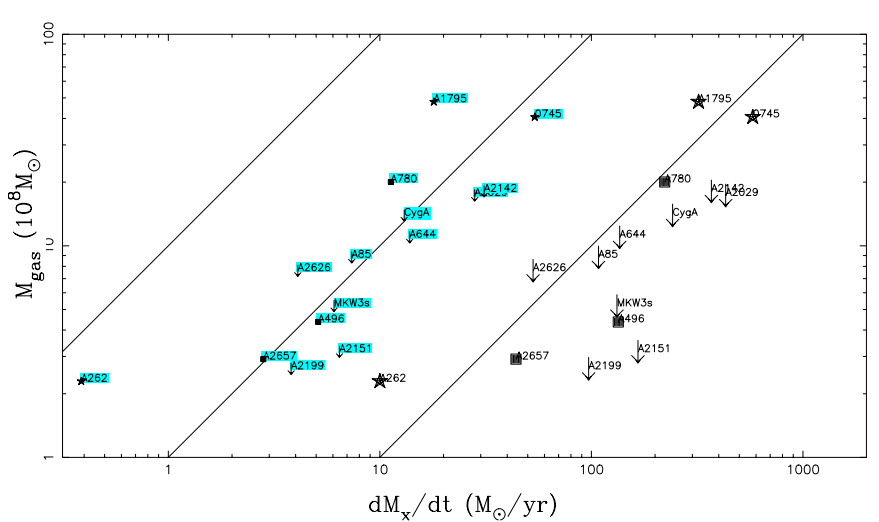

Fig. 3. $M_{\text {gas }}$ from CO observations with respect to $\dot{M}$ the mass deposition rates deduced from Einstein X-ray data by White et al. (1997). Straight lines from right to left are for $M_{\mathrm{gas}}=1 \% \times \dot{M} \times 1 \mathrm{Gyr}$, $M_{\mathrm{gas}}=10 \% \times \dot{M} \times 1 \mathrm{Gyr}$ and $M_{\mathrm{gas}}=\dot{M} \times 1 \mathrm{Gyr}$.

identically in all clusters of galaxies centers, depending on the environment of the cooling flow (existence or not of an AGN for example). 


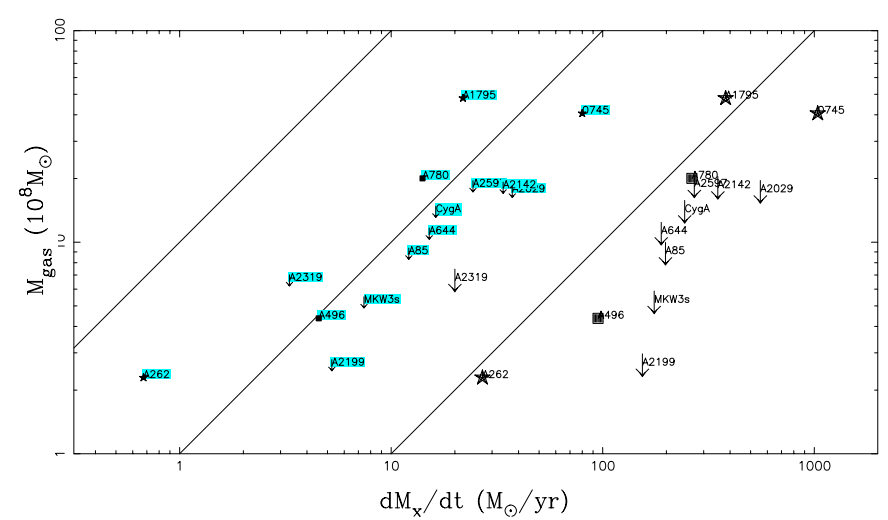

Fig. 4. $M_{\text {gas }}$ from CO observations with respect to $\dot{M}$ the mass deposition rates deduced from ROSAT X-ray data by Peres et al. (1998).

\subsection{Heating by $A G N$}

Many studies developed recently are taking into account heating mechanisms in cooling flows that could slow down the cooling and eventually stop it, which might explain the lack of $\mathrm{CO}$ emission in some of the galaxies observed here. Important absorption could also hide the gas lying in a colder X-ray phase. Physical conditions in the central regions are certainly very complex, and simple cooling appears to be insufficient to explain multi-wavelength observations. Chandra images from Fabian et al. (2002) or Johnstone et al. (2002) have pointed out holes coincident with radio lobes and cold fronts showing the interaction between the radio source and the intra-cluster medium (e.g. in Hydra A, the Perseus cluster, Abell 1795, Abell 2199, the Virgo cluster). It seems that the radio source and jets could heat the gas with shocks and significantly decrease the cooling rates (David et al. 2001; Brüggen et al. 2002). Besides, $71 \%$ of central $\mathrm{cD}$ galaxy in cooling flow clusters show a strong radio activity compared to $23 \%$ for non-cooling flow cluster cDs (Ball et al. 1993). No correlation has been found here between molecular gas masses and radio power at $1.4 \mathrm{GHz}$ (see Fig. 5). Nevertheless, it seems that for faint radio sources, the power at $1.4 \mathrm{GHz}$ increases with the cold molecular gas mass detected, and for stronger radio sources, $M_{\text {gas }}$ decreases when the radio power increases. This is consistent with a self-regulated heating model powered by a central AGN (as suggested by Böhringer et al. 2001). But heating the ICM is certainly due to the radio lobes expansion whose energy is not only linked to the radio power.

\subsection{Correlations with $H_{\alpha}$ and $M_{\text {dust }}$}

The molecular hydrogen mass appears to be correlated with the amount of ionized gas (see Fig. 6). Excitation processes leading to the gas emission could be the same for the two phases. The $\mathrm{H} \alpha$ emission could come from shocked cooling gas. The gas may also have been ionized from massive stars born in a starburst triggered by the cooling flow. The best linear fit between the two components is plotted including previous detections of Edge (2001), (Fig. 6). Heating by a young star population is often suggested (see Johnstone et al. 2002). In that sense, gradients of metallicity deduced from Chandra observations could

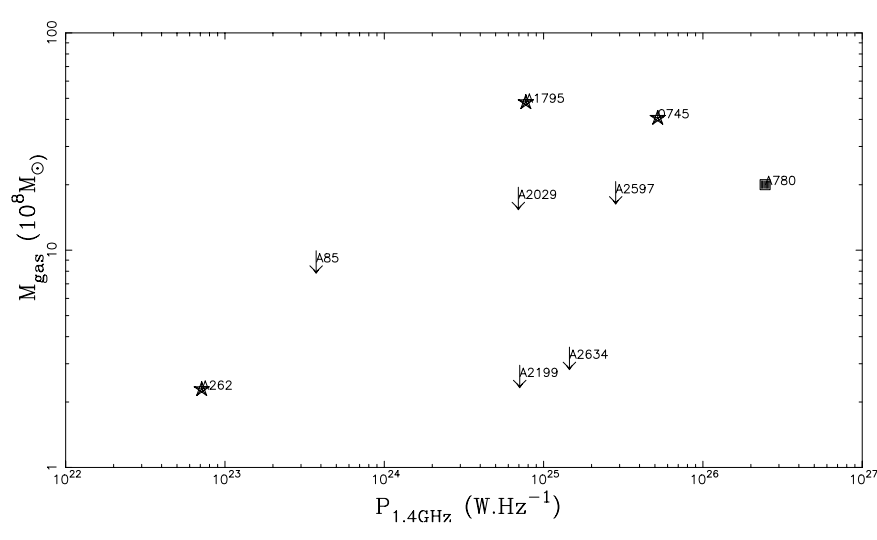

Fig. 5. $M_{\text {gas }}$ versus radio power at $1.4 \mathrm{GHz}$. There is no clear correlation between molecular gas masses and radio flux.

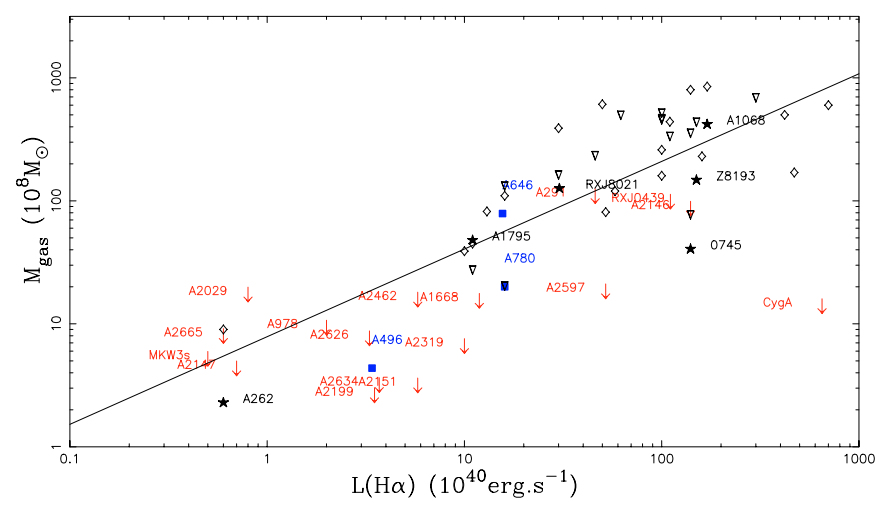

Fig. 6. $M_{\text {gas }}$ deduced from our $\mathrm{CO}$ observations versus $\mathrm{H}_{\alpha}$. The straight line here is a fit to the detection and hint of detection points. Data from Edge (2001) are plotted. Diamond shaped are the detections and triangles are the upper limits. Upper limits were not taken into account in the fit. There is a clear correlation between $\mathrm{H}_{\alpha}$ luminosity and cold gas masses, in agreement with previous detections.

be explained by SN Ia injection of metal in the central galaxy (with the condition of some exchange of the gas at different radius, and so a possible mixing of different phases of the gas if they are present). The cold gas detected here might be a reservoir available for such a star formation process.

The intracluster gas should be depleted in dust, at a given metallicity, since in the ICM environment and its physical conditions, the sputtering time of dust is much shorter than the dynamical time. The gas coming from a cooling flow, already at low metallicity, is thought to have a large relative depletion in dust. Therefore, the expected ratio between the $\mathrm{CO}$ measured gas content and the dust content from its submillimeter or farinfrared emission is large. Dust masses, derived from IRAS are evaluated for two assumed dust temperatures $T_{\text {dust }}=35$ and $40 \mathrm{~K}$. Theses masses are compared to cold gas masses in Fig. 7. It is important to notice how much dust mass highly depends on dust temperature. The gas-to-dust ratio for both dust temperature are high, but below $\sim 2 \times 10^{3}$. However, only IRAS data have been used here, tracing the warm dust. Significant amount of cold dust might be present as suggested by JCMT SCUBA detections in Abell 1835, Abell 2390 (Edge et al. 1999). More longer wavelength observations at $850 \mu \mathrm{m}$ tracing this cool dust would be of great interest. Nevertheless the mass to dust ratio 

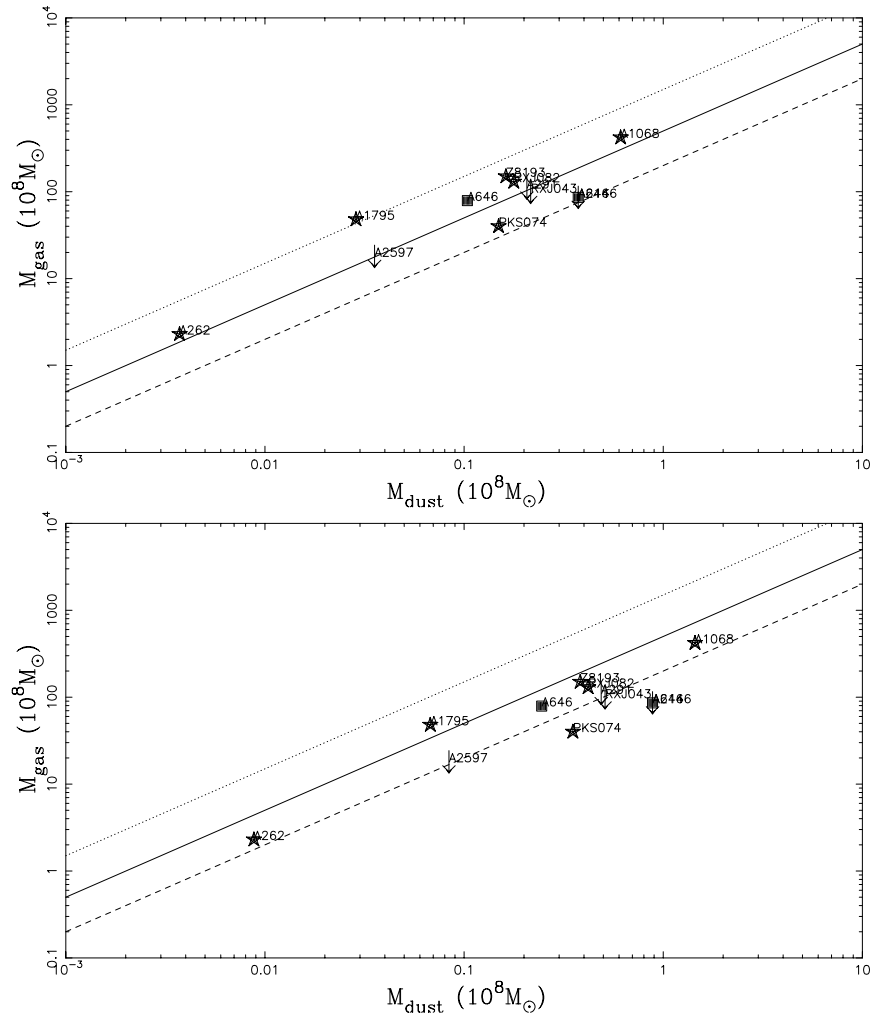

Fig. 7. $M_{\text {gas }}$ molecular hydrogen mass given by $\mathrm{CO}$ measurements as a function of $M_{\text {dust }}$ obtained from IRAS data. The dust mass highly depends on the dust temperature which is unknown: the dust temperature is estimated to $35 \mathrm{~K}$ on the upper figure and to $40 \mathrm{~K}$ on the figure below. The straight lines represent gas to dust ratio of 200 (dashed), 500 (full) and 1500 (dotted). The temperature uncertainties exclude any clear conclusion about the cold gas origin.

found here are not incompatible with a cooling flow origin of the molecular gas. Besides, there is a trend of correlation between cold gas masses and dust masses, but with a large dispersion. Infrared emission might be tied to star formation. In that sense, Fig. 7 could also be interpreted as a possible correlation between gas content and star formation, probably very active, as we have seen previously.

\subsection{Excited $\mathrm{H}_{2}$ detected in NIR}

Some of our sample galaxies have strong molecular hydrogen emission in the $2 \mu \mathrm{m} \mathrm{1-0} \mathrm{S(1)} \mathrm{line,} \mathrm{(Elston} \mathrm{\&} \mathrm{Maloney} \mathrm{1992,}$ 1994). This excited gas is thought to be associated to the cooling flow, since it is not detected in non-cooling flow galaxies of similar-type (Jaffe \& Bremer 1997). They reveal dusty nebular filaments, very similar to those detected in early type galaxies in small groups e.g. Goudfrooij \& Trinchieri (1998), and in interacting gas-rich galaxies. The filaments are extended over kiloparsecs, and their heating source is not known.

Recently, Donahue et al. (2000) have mapped the kpc-size filaments in vibrationally-excited $\mathrm{H}_{2}$ in the cores of galaxies centers of Abell 2597 and PKS 0745-191 with high spatial resolution. They have also found dust lanes which are optically thick to $1.6 \mu \mathrm{m}$ emission, confined to the central few kpcs. Excited $\mathrm{H}_{2}$ produced directly by the cooling flow seems difficult, since $\mathrm{H}_{2}$ is much too luminous, by at least 2 orders of magnitude. It cannot be AGN photoionization or fast shocks because the $\mathrm{H} \alpha / \mathrm{H}_{2}$ ratios are too low. Extremely slow shocks $\left(<40 \mathrm{~km} \mathrm{~s}^{-1}\right)$ produce significantly higher $\mathrm{H}_{2} / \mathrm{H} \alpha$ ratios than do fast shocks, and are more consistent with the observations. But slow shocks are less efficient. The most likely solution is UV irradiation by very hot stars, implied by a star formation rate of only a few solar masses per year. A recent survey of $H$-band and $K$-band spectra in 32 central cluster galaxies have led to 23 detections in rovibrationnal $\mathrm{H}_{2}$ lines, see Edge et al. (2002), and UV fluorescence excitation is ruled out. The molecular hydrogen is more probably thermally excited in dense gas with density exceeding $10^{5} \mathrm{~cm}^{-3}$ at temperature evaluated between $1000-2500 \mathrm{~K}$. Young stars heating a population of dense clouds is invoked (Wilman et al. 2002). According to the authors, these dense regions might be self gravitating clouds deposited directly by the cooling flow or confined in high pressure behind strong shocks. Correlation are also shown between $\mathrm{H}_{\alpha}$ emission lines, warm $\mathrm{H}_{2}$ rovibrationnal lines and cold millimetric emission lines suggesting related exciting mechanisms of these different phases of the gas. The large masses of excited $\mathrm{H}_{2}$, around $10^{5-6} M_{\odot}$ could suggest that the cold molecular gas mass could have been underestimated (because of a lower metallicity for example) or is hidden in optically thick dense clouds, see Ferland et al. (2002) for a discussion of the physical conditions within dense cold clouds in cooling flows.

How much gas is deposited in cooling flows is still an open question. The gas cooling in the flow is probably multiphase, and there are hints the $\mathrm{CO}$ detected here is the residual of the cooled gas. But this cold gas emission could also be due to subcluster structures, gas stripped from neighbouring galaxies or galactic clouds not seen until now and heated by mechanisms linked to the flow, like shocks or starburst. More investigations are required to explore the properties of this important component in cooling flow cluster cores. The study of the morphological structure of the cold gas and especially its dynamics will help to confirm its place in the flow. High resolution maps, obtained thanks to the IRAM millimeter interferometer, have been obtained for Abell 1795 in $\mathrm{CO}(1-0)$ and $\mathrm{CO}(2-$ 1). These maps show an extended emission of the cold gas (Salomé \& Combes, in prep). They underline the possible link between the cold gas detected with the $30 \mathrm{~m}$ telescope and the cooling gas seen at higher energy. Recent OVRO observations by Edge \& Frayer (2003) also show CO(1-0) emission maps in 5 cooling flow clusters of galaxies: A1068, RX J0821+07, Zw3146, A1835 and RX J0338+09. The authors conclude the gas previously detected with the single dish telescope is confined in the central region. More Plateau de Bure interferometric observations with higher sensitivity and spatial resolution are in progress now in RXJ0821+07 to see whether the cold gas is extended (as for Abell 1795) or centrally concentrated around the cD (as suggest the OVRO observations). Interferometric observations on a wider sample of $\mathrm{CO}$ detected cooling flow have now to be lead in order to explore the similarities and differences between clusters and definitively confirm the detection of the cold residual in cooling flows. 


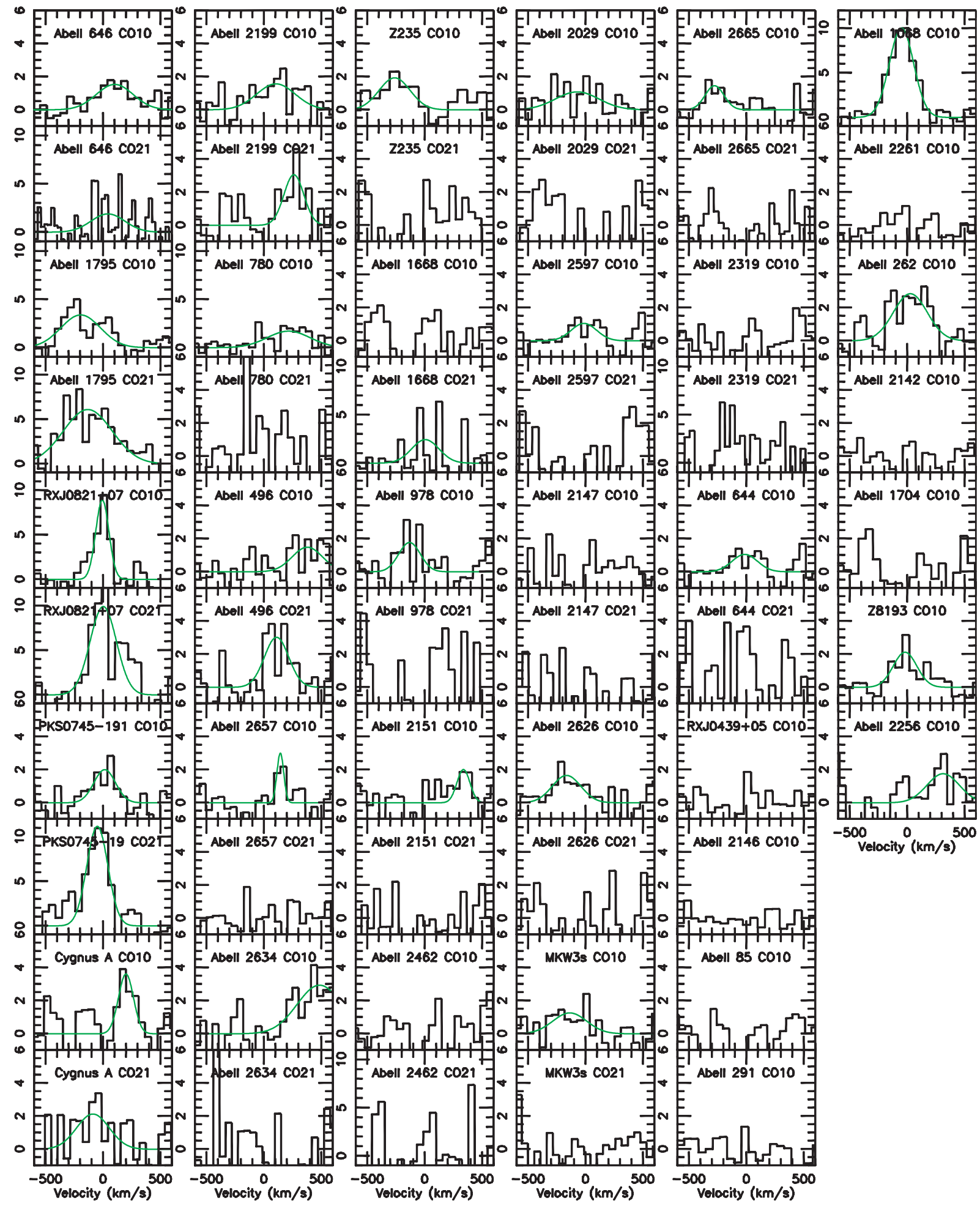

Fig. 8. $\mathrm{CO}(1-0)$ and $\mathrm{CO}(2-1)$ emission lines observed with the IRAM $30 \mathrm{~m}$ telescope. On the $Y$-axis, main beam temperature (in $\mathrm{mK}$ ) versus velocity (in $\mathrm{km} \mathrm{s}^{-1}$ ) on the $X$-axis.

\section{Conclusions}

A sample of 32 cooling flow clusters of galaxies, selected on their mass deposition rate, and their $\mathrm{H} \alpha$ luminosity, have been observed in both $\mathrm{CO}(1-0)$ and $\mathrm{CO}(2-1)$ emission lines. In total 6 clear detections are claimed, with 4 other possible detections.
Molecular hydrogen mass estimates have been deduced for these galaxies and upper limits have been computed for the other ones. The derived $M\left(\mathrm{H}_{2}\right)$ are up to $10^{10} M_{\odot}$ in the 22 central arcsec observed with the $30 \mathrm{~m}$ telescope (that is typically the central $\sim 23 \mathrm{kpc}$ region at $z=0.05$ ). These masses appear to be related to the cooling rate deduced from X-ray data: there is 
a trend of correlation with $\dot{M}_{\mathrm{X}}$ results, and no longer large discrepancies between the mass deposition rates and the cold gas masses (according to recent mass deposition rates reevaluation from Chandra and XMM-Newton). The apparent gas-to-dust ratio, derived from the $\mathrm{CO}$ emission and dust far-infrared emission is larger for the gas in cooling flow galaxies than in normal spirals, but uncertainties about the dust temperature precludes any clear conclusions. The best correlation is between the cold gas masses and the $\mathrm{H} \alpha$ luminosities, which confirms the result of Edge (2001). Further work is to be done now to confirm that CO lines, revealed by single dish millimetric observations, are tracers of the long searched cold phase in cooling flows. In this context, more interferometric observations in $\mathrm{CO}(1-0)$ and $\mathrm{CO}(2-1)$ are required.

Acknowledgements. It is a pleasure to thank the IRAM-30 m staff for their support during observations and data reduction, especially with the new $4 \mathrm{MHz}$ filter-bank. We also thank A. Edge for his constructive refereing.

\section{References}

Antonucci, R., \& Barvainis, R. 1994, AJ, 107, 448

Allen, S. W. 2000, MNRAS, 315, 269

Baum, S. A., \& O'Dea, C. P. 1991, MNRAS, 250, 737B

Bayer-Kim, C. M., Crawford, C. S., Allen, S. W., Edge, A. C., \& Fabian, A. C. 2002 MNRAS, 337, 938

Bonamente, M., Lieu, R., \& Mittaz, J. P. D. 2001, ApJ, 546, 805

Bonamente, M., Lieu, R., \& Mittaz, J. P. D. 2001, ApJ, 547, L7

Böhringer, H., Matsushita, K., \& Ikebe, Y. 2001, [astro-ph/0111113]

Braine, J., \& Combes, F. 1992, A\&A, 264, 433

Braine, J., \& Dupraz, C. 1994, A\&A, 283, 407

Bregman, J., Mc Namara, B. R., \& O’Connell, W. 1990, ApJ, 351, 406

Brighenti, F., \& Mathews, W. G. 2002 [astro-ph/0203409]

Brüggen, M., Kaiser, C. R., Churazov, E., \& Ensslin, T. A. 2002, MNRAS, 331, 545B

Burns, J. O., White, R. A., \& Haynes, M. P. 1981, AJ, 86, 1120

Ball, R., Burns, J. O., \& Loken, C. 1993, AJ, 105, 53

Cowie, L. L., Hu, E. M., Jenkins, E. B., \& York, D. G. 1983, ApJ, 272, $29 \mathrm{C}$

Crawford, C. S., Allen, S. W., Ebeling, H., Edge, A. C, \& Fabian, A. C. 1999, MNRAS, 306, 857

David, L. P., Slyz, A., Forman, W, \& Vrtilek, S. D. 1993, ApJ, 412, 479

David, L. P., Nulsen, P. E. J., McNamara, B. R., et al. 2001, ApJ, 557, 546

Donahue, M., Mack, J., Voit, G. M., et al. 2000, ApJ, 545, 670

Dunne, L., \& Eales, S. A. 2001, MNRAS, 327, 697

Dupke, R., \& White III R. E. 2003, ApJ, 583, L13

Durret, F., Gerbal, D., Lachièze-Rey, M., Lima-Neto, G., \& Sadat, R. 1994, A\&A, 287, 733

Dwarakanath, K. S., Owen, F. N., \& van Gorkom, J. H. 1995, ApJ, 442, L1
Edge, A. C., Ivison, R. J., Smail, Ian, Blain, A. W., \& Kneib, J. P. 1999, MNRAS, 306, 599

Edge, A. C. 2001, MNRAS, 328, 762

Edge, A. C., Wilman, R. J., Johnstone, R. M., et al. 2002, [astro-ph/0206379]

Edge, A. C., \& Frayer, D. T. 2003, ApJ, 594, L13

Elston, R., \& Maloney, P. 1992, BAAS, 181, 118.11

Elston, R., \& Maloney, P. 1994, in Infrared Astronomy with Arrays: The Next Generation, ed. I. S. McLean (Dordrecht: Kluwer), 169

Ettori, S., Fabian, A. C., Allen, S. W., \& Johnstone, R. M. 2002, MNRAS, 331, 635E

Fabian, A. C. 1994, ARA\&A, 32, 277

Fabian, A. C., Sanders, J. S., Ettori, S., et al. 2001, MNRAS, 321, L33

Fabian, A. C. 2002 [astro-ph/0201386]

Fabian, A. C., Celotti, A., Blundell, K. M., Kassim, N. E., \& Perley, R. A. 2002, MNRAS, 331, 369

Falcke, H., Rieke, M. J., Rieke, G. H., Simpson, C., \& Wilson, A. S., 1998, ApJ, 494, L155

Ferland, G. J., Fabian, A. C., \& Johnstone, R. M., 1994, MNRAS, 266, 399

Ferland, G. J., Fabian, A. C., \& Johnstone, R. M. 2002, [astro-ph/0203052]

Grabelsky, D. A., \& Ulmer, M. P. 1990, ApJ, 355, 401

Goudfrooij, P., \& Trinchieri, G. 1998, A\&A, 330, 123

Jaffe, W., \& Bremer, M. N. 1997, MNRAS, 284, L1

Johnstone, R. M., Allen, S. W., Fabian, A. C., \& Sanders, J. S. 2002, [astro-ph/020207]

Lazareff, B., Castets, A., Kim, D. W., \& Jura, M. 1989, ApJ, 335, L13

Lieu, R., Mittaz, J. P. D., Bowyer, S., et al. 1996, ApJ, 458, L5

Lieu, R., Bonamente, M., \& Mittaz, J. P. D. 1999, ApJ, 517, L91

McNamara, B. R., Bregman, J. N., \& O'Connell, R. W. 1990, ApJ, 360, 20

McNamara, B. R., \& Jaffe, W. 1994, A\&A, 281, 673

McNamara, B. R. 2002 [astro-ph/0202199]

Mittaz, J. P. D, Lieu, R., \& Lockman, F. J. 1998, ApJ, 498, L17

O’Dea, C. P., Baum, S. A., \& Gallimore, J. F. 1994b, ApJ, 436, 669

O’Dea, C. P., Baum, S. A., Maloney, P. R., Tacconi, L. J., \& Sparks, W. B., ApJ, 422, 467

Owen, F., Ledlow, M. J., \& Keel, W. C. 1995, AJ, 109, 14

Peres, C. B., Fabian, A. C., Edge, A. C., et al. 1998, MNRAS, 298, 416

Peterson, J. R., Paerels, F. B. S., Kaastra, J. S., et al. 2001, A\&A, 365, L104

Pfenniger, D., \& Combes, F. 1994, A\&A, 285, 94

Shostak, G. S., van Gorkom, J. H., Ekers R. D., et al. 1983, A\&A, 119, L3

Tamura, T., Kaastra, J. S., Peterson, J. R., et al. 2001, A\&A, 365, L87

Tucker, W., \& David, L. P. 1997, ApJ, 484, 602

Valentijn, E. A., \& Giovanelli, R. 1982, A\&A, 114, 208

Voigt, L. M., Schmidt, R. W., Fabian, A. C., Allen, S. W., \& Johnstone, R. M. 2002 [astro-ph/0203312]

White, D. A., Jones, C., \& Forman, W. 1997, MNRAS, 292, 419

Wilman, R. J., Edge, A. C., Johnstone, R. M., et al. [astro-ph/0206382]

Wu, X. P., \& Hammer, F. 1993, MNRAS, 262, 187 